\title{
Strategies and Challenges to Facilitate Situated Learning in Virtual Worlds Post-Second Life
}

\author{
MARC CONRAD, ADIL HASSAN, LYZGEO KOSHY, ASLAN KANAMGOTOV, \\ and ATHANASIOS CHRISTOPOULOS, Department of Computer Science and Technology, \\ University of Bedfordshire, United Kingdom
}

\begin{abstract}
Virtual worlds can establish a stimulating environment to support a situated learning approach in which students simulate a task within a safe environment. While in previous years Second Life played a major role in providing such a virtual environment, there are now more and more alternative-often OpenSimbased-solutions deployed within the educational community. By drawing parallels to social networks, we discuss two aspects: how to link individually hosted virtual worlds together in order to implement context for immersion and how to identify and avoid "fake" avatars so people behind these avatars can be held accountable for their actions.
\end{abstract}

CCS Concepts: - Human-centered computing $\rightarrow$ Virtual reality; $\quad$ Computer systems organization $\rightarrow$ Peer-to-peer architectures

Additional Key Words and Phrases: Virtual worlds, social networks, situated learning, identity

ACM Reference Format:

Marc Conrad, Adil Hassan, Lyzgeo Koshy, Aslan Kanamgotov, and Athanasios Christopoulos. 2017. Strategies and challenges to facilitate situated learning in virtual worlds post-second life. Comput. Entertain. 15, 1, Article 3 (February 2017), 9 pages.

DOI: http://dx.doi.org/10.1145/3010078

\section{INTRODUCTION}

The "situated learning" approach by Herrington and Oliver [2000] encompasses parameters such as authentic context and multiple roles as well as provides perspective and collaborative construction of knowledge. With the increase (and even more so with the recent "hype") on virtual worlds, these can be identified as a means to provide an "authentic context" in which a situated learning approach can take place.

An-at least often perceived-advantage of virtual worlds is that they provide an experience of "reality" in an otherwise safe and controllable environment. However, there is an inherent tradeoff in this: The more realistic the simulated environment, the more there is a danger that this environment is then, in addition to the positive benefits, as well affected negatively by the behavior of its population. An issue here is played by the traceability of the avatar to a real person that can be held accountable for his or her actions.

A further aspect requiring attention is a potential dependency on a central provider such as Second Life. As a partial response to this situation, many virtual world projects nowadays use local, OpenSim-based solutions as an alternative. However, to provide

Authors' addresses: M. Conrad, A. Hassan, L. Koshy, A. Kanamgotov, and A. Christopoulos, University of Bedfordshire, Park Square, Luton, LU1 3JU; emails: \{Marc.Conrad, ADIL.HASSAN, lyzgeo.koshy, Aslan.Kanamgotov\}@beds.ac.uk.

Permission to make digital or hard copies of part or all of this work for personal or classroom use is granted without fee provided that copies are not made or distributed for profit or commercial advantage and that copies show this notice on the first page or initial screen of a display along with the full citation. Copyrights for components of this work owned by others than ACM must be honored. Abstracting with credit is permitted. To copy otherwise, to republish, to post on servers, to redistribute to lists, or to use any component of this work in other works requires prior specific permission and/or a fee. Permissions may be requested from Publications Dept., ACM, Inc., 2 Penn Plaza, Suite 701, New York, NY 10121-0701 USA, fax +1 (212) 869-0481, or permissions@acm.org.

(c) 2017 ACM 1544-3574/2017/02-ART3 $\$ 15.00$

DOI: http://dx.doi.org/10.1145/3010078 
context in order to facilitate a better immersion they would ultimately benefit from being linked together.

Both aspects of virtual worlds-accountability of the avatar and decentralized service provision-are mirrored within the domain of social networks: one provider (Facebook) has a dominant role, and "fake" accounts exist as well. In this article, we investigate the situation within social networks with the goal to transfer the lessons learned there to the case of virtual environments.

The article is organized as follows: first, as a means of illustration, we describe how virtual worlds have been used at the University of Bedfordshire to enhance the delivery of Project Management as a topic. A prerequisite for this is the presence of immersion, which raises questions given the multitude of devices that can be used to access these devices and the environment they are operated in-this is further discussed in Section 3. However, it is not only the external environment but also the in-world context that contributes to immersion. The nature of this relationship is investigated in Section 4. The provision of this immersion within the context of an educational institution is a challenge: To provide context, a decentralized structure would be preferable, where each university keeps responsibility of their own students' data while still being connected. Approaches to setup such a decentralized structure are evaluated in Section 5. Last but not least, such a distributed approach leads to the question of security. In particular, while in an online role-playing environment a certain extent of anonymity is acceptable or can be seen as a feature. This is less likely the case within an educational context, which is discussed in Section 6 . The final conclusion draws these findings together.

\section{CASE STUDY: PROJECT MANAGEMENT}

Project Management is an inherently inter-disciplinary activity and defined by the Project Management Institute as "the application of knowledge, skill, tools, and techniques to project activities to meet project requirements" [PMI 2008]. In order to teach Project Management, an approach based on practical, hands-on experience seems most appropriate. While some knowledge areas of Project Management are straightforward to deliver, for instance, time management is enforced by the inherent context of a university assignment that is linked to "natural" deadlines; other areas, such as Risk or Procurement, are less obvious to be incorporated.

Indeed, health and safety considerations as well as common sense suggest that students should not be exposed to a source of "real" project risks such as damage to health, bankruptcy, or other material loss. The use of Virtual Worlds-as these provide a controlled environment-is therefore a useful way to implement risk within a project management course.

Virtual worlds have been used within teaching of Project Management at the University of Bedfordshire since 2008. The more experimental experience within Second Life (Figure 1) as a provider is documented in Conrad et al. [2009]. A systematic exposition of Second Life is then detailed within Conrad [2011a]. In 2011, a different provider, Reaction Grid, has been used (Figure 2). While the underpinning technology is similar, there have been notable differences between these two environments [Kanamgotov et al. 2012]: a lack of immersion within the much lesser populated world of Reaction Grid raises questions on how such activities need to be designed to be successful.

\section{IMMERSION}

As seen in the previous section, three-dimensional (3D) virtual realities can greatly enhance the learning experience by bringing new technologies and approaches in onlinebased education, providing a richer environment, more resembling the real classroom and allowing for deeper involvement-via immersion—of students. Currently, many 


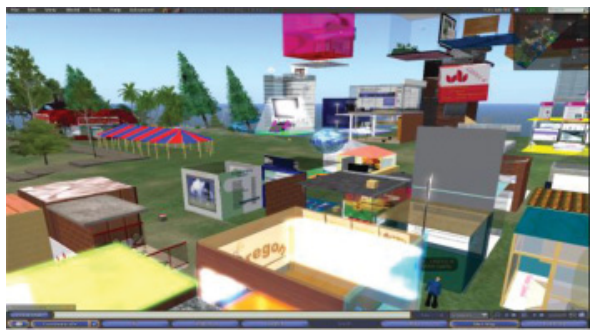

Fig. 1. Second Life, 2008.

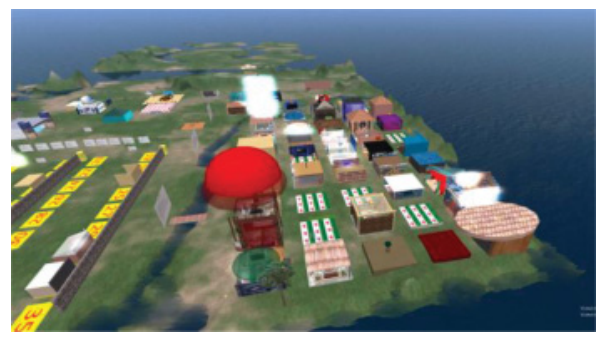

Fig. 2. Reaction Grid, 2012.

universities utilize the tablet PC in formal taught courses [Moore et al. 2008], allowing students to revise material at their convenience. Does this new technology enhance the learning process, or, in contrast, does it limit the learner's ability to comprehend material? With reference to the characterization of immersion by Paush et al. [1997], will the 3D environment, being accessed from a tablet PC, provide the same level of "immersion" or the sense of "being there"? One of the aims is therefore to quantify immersion in Multiuser Virtual Realities (MVR).

In 2007, the University of Bedfordshire began to utilize Second Life for classes in the Linden Scripting Language and Project Management. In a previous experiment, which was already the continuation of earlier research [Conrad 2011a], an attempt was made to quantify immersion in a virtual reality environment that was not Second Life but situated within the space rented from a dedicated provider (namely Reaction Grid). Students were required to build an educational showcase within this and to develop an artifact of their own choice (unrelated to virtual worlds). Results indicated that not much immersion happened in this scenario. The reason for the lack of immersion experience was identified in the lack of communication due to a general underpopulation of this virtual world used for experiment [Kanamgotov et al. 2012].

In further research, we aim to measure immersion in an MVR that is used for educational purposes. We argue that immersion, which plays a vital role in material comprehension, will not be lost due to technical constraints of a tablet PC (for example, smaller screen size, lower processing power). Having the tablet as the only means of communication while being out of class or home, as we believe, brings more sense of concentration and immersion into material.

Previous experiments [Cox et al. 2006] have been undertaken within non-mobile VR environments and the user's goal was clearly identified to find hidden objects or to play predefined games. In games or, for instance, training simulators the most immersed users, as indicated by the experiments' results, demonstrated better performance [Bowman et al. 2007]. However, there is also the "less is more" approach. Gaps and omissions stimulate engagement since the reader fills the gaps of what happens between each panel of the illustrated story [McCloud 1994]. But how much immersion would be enough and how that affects the comprehension of the material [Carr and Oliver 2009]?

Therefore, we seek to investigate in a structured experiment the influence of mobility on immersion experience while using a Tablet PC for learning course material within MVR. The aim of this project is to create a novel methodology or framework to quantify immersion. Participants will be doing a "task" in MVR. By "task" we mean the presence in MVR without a clearly identified set of goals. An example for this might be a project team meeting (in contrast to strictly goal-oriented game environment). During the experiment, participants must be isolated from each other, physically being in different places, to prevent "real life" communication. All the communication must be 
held exclusively through avatars in MVR. It is expected that results will demonstrate that mobile immersion will not significantly drop in comparison to stationary environment. The reason for this conclusion lies in the results of our previous research, which indicated that immersion mostly depends on successful communication among users rather than technical aspects.

\section{CONTEXT DEFINES IMMERSION}

In our experiments, we also identified the underpopulation of MVR's as a contributing factor in the lack of immersion encountered within a virtual world. As part of a wider study -in the context of a Masters by Research project, with partial results published in Christopoulos and Conrad [2012] — that evaluates the validity of the framework introduced by Conrad [2011b], the relevance of context within a virtual world has been explored and its particular impact towards the educational aims of three activities. The features of Second Life and OpenSim have been investigated to identify the rationales in educators' choices to use a Virtual World of one kind or another in order to meet the learning needs of their students.

For the conduct of that research study, what was taken into account is a pre-existing framework that proposes the evaluation of virtual worlds against four dimensions: their contexts, the immersion encountered within each, their cost, and their persistence [Conrad 2011b]. The findings indeed validated this with some alterations and highlight the perceived and real similarities and differences between Second Life and OpenSim worlds: None of these options can be considered as "the best." On the contrary, the answer to the question "which is the ideal virtual world for the conduct of educational projects?" depends very centrally on each educator's particular needs. Nevertheless, it is possible to provide clear guidance to academics to face such a decision. In particular, in many cases, OpenSim-based worlds provide a viable alternative to a Second Lifebased environment.

OpenSim-based worlds come in two major categories: They can be hosted by an external provider or they can be hosted by the institution itself. The latter case raises important questions on how these OpenSim-based worlds of different educational institutions can be linked together to provide a perception of a "world" with context within.

\section{DISTRIBUTED APPROACHES}

One example of a distributed OpenSim-based world is the OSGrid (http://www. osgrid.org). To identify further examples of different approaches to link virtual environments, we consider a different domain that is the domain of online social networks (OSN's). Distributed visual environments (DVE) indeed have similarities with online social networks: Both allow the users in a network to interact with each other by sharing common interests. Also, issues such as scalability, communication architecture, replication, and concurrency control are common between DVE's and OSN's. Lee et. al [2002] pointed out a number of issues associated with the DVE's that is, scalability, communication architecture, replication, and concurrency control. Decentralized architectures in online social networks have similar issues with managing data, privacy, security, and data replication to those being encountered in DVEs.

The success of OSN's has changed the way people interact and communicate with each other. Among the most dominant social networks are Facebook, Twitter, and MySpace. These and other OSN's have indeed had a great influence on businesses by offering new and interactive ways to advertise their products or services online and to reach potential customers. Yeung et al. [2009] claim that where the OSN's have brought advantages to the community and businesses it also has put the users' privacy and security at risk. 
There are a number of issues associated with the existing design of social network service providers. Some of the key problems are as follows: (i) Users do not have control over their data [Yeung et al. 2009; Bielenberg et al. 2012; Seong et al. 2010]; (ii) users have to agree to the terms and conditions of the service providers, which can be changed anytime without any prior notice [Bielenberg et al. 2012; Fischer et al. 2010]; (iii) there is no set of fine-grained access control policies [Bielenberg et al. 2012]; (iv) private information may be leaked (Yeung et al. [2009] report that some of the service providers were being caught selling user data illegally to third parties); and (v) businesses advertising their products and services on OSN's to attract customers might encounter privacy leakages in terms of their relationships with the customers. This has raised privacy concerns among the users and in the research community [Bielenberg et al. 2012; Krishnamurthy and Wills 2009].

Most of the existing social networks, for example, Facebook, Twitter, and MySpace, are built on centralized architectures. That means that users on these and other centralized social networks are administered by a single domain and they have full control over their data. Decentralized models of social networks allow data to be distributed among different administrative domains that users' trust, including their own server. This gives users ownership of their data and allows them to tailor their data sharing policies to their exact needs.

For these reasons, alternative solutions have been proposed and many attempts have been made to create a semi/fully decentralized model of OSNs' where the users can have control over their data and should have fine-grained access control policies. Several proposals in the literature have been made that aim to provide better users privacy by decentralizing the existing architecture of OSNs. The three systems of semi/fully decentralized OSNs we investigate here are Diaspora, Mr. Privacy, and PeerSon; among these, Diaspora and Mr. Privacy are the only large-scale existing online active social networks [Bielenberg et al. 2012; Fischer et al. 2010], whereas PeerSon is built as a prototype and is still in development stages.

In the section below, we will review these systems that attempt to create a decentralized architecture of OSN's in more detail.

Diaspora. Diaspora was built on a semi-decentralized architecture using push design to send data and notifications to other servers and Uniform Resource Identifiers (URIs') as WebID's that were used as reference/pointer to a source of information/users. Diaspora preserves the users' privacy by allowing them to define their access control polices, similarly to the "circles" introduced by google+ [Bielenberg et al. 2012]. Because of the privacy-preserving design of Diaspora, it allows its users to choose on which server they want to host their profile. They can also administer their profile on their own server. One of the problems with the architecture of Diaspora was that users have to trust their friends' server, as a replica of their user profile sits on their friends' server as well. Another problem is that communication between servers is not encrypted. But Bielenberg et al. [2012] also claim that this can be resolved in a straightforward way by encrypting user data before it is routed on the Diaspora network/servers. Another problem is that for the successful transaction of communication between the users, they need to be online at the same time, as otherwise messages get lost in the network and the sender does not get any acknowledgement; thus highlighting the issue of synchronization as a central problem to focus on in a decentralized environment where data replication is the norm rather than the exception.

Mr. Privacy. Fischer et al. [2010] have proposed an open and federated social networking experience built on Email and OpenID. Email was used primarily as a distributed personal database that holds users' information. The model uses Simple Mail Transfer Protocol to send emails and Internet Message Access Protocol (IMAP) to retrieve 
emails from the server. Fischer et al. [2010] claim that the success of Mr. Privacy lies in it being inter-operable insofar that users do not have to join any particular social network, and it saves them from sending/accepting or rejecting friend requests. It allows the users to share information without repercussions; the defining feature-email infrastructure-is, however, as well a problem: In using the existing email infrastructure, it uses this system both as a queue for messages and as an implicit database. This implies questions on the ownership of the users' data. Fischer et al. [2010] also identified some technical issues associated with this model as well, such as the lack of universal support for IMAP as it is compatible only with Gmail and AOL but not with Yahoo and Hotmail, among others.

Peerson. Buchegger et al. [2009] propose a model using Peer-to-Peer (P2P) infrastructure. Apart from its decentralized architecture, PeerSon also implements encryption ensuring privacy in social networks and direct data exchange that allows users to use a system without constant internet activity. PeerSon uses OpenDHT-a Distributed Hash Table- (DHT) type infrastructure as a look-up service that provides a mechanism to keep nodes connected as necessary. Buchegger et al. [2009] highlight a number of issues associated with DHT, that is, storage limitations, security, availability, and down times. The prototype of PeerSon was successful on a small scale but they have encountered a number of problems related to the DHT approach.

We have seen different approaches to create a semi/fully decentralized architecture. All of these have gone to the prototype stage or, in the case of Diaspora, been implemented albeit without the success of a widespread adaption by a target user group.

The architectures range from a multi-server approach, as seen in Diaspora over utilizing an existing infrastructure (emails in Mr. Privacy), to a fully peer-to-peer-based system, as suggested in Peerson. Translated to virtual worlds, it shows that alternatives to the OSGrid structure (which in its basic principles resembles the structure found in Diaspora-groups of users on a number of independent servers) may be possible but then create different issues to be resolved.

As seen above, there are a number of issues associated with decentralizing the architectures of online social networks that are also recognizable in the context of DVEs. The most important issues are scalability, data privacy, integrity, and users' availability. The main strategies to resolve this are efficient data replication with concurrency control and interest management and by implementing self-healing processes for a fault-tolerant communication architecture [Lee et al. 2002].

\section{IDENTITIES IN VIRTUAL ENVIRONMENTS}

As reported by Christopoulos and Conrad [2012], the coexistence of users and their interaction with others in the context of the virtual world is a factor identified to experience immersion. However, implied by this co-existence, a certain concern to educators is a possible disturbance to educational activities from other residents within this world who deliberately intervene negatively with other avatars, so-called griefers. A prerequisite of such behavior is the existence of a "fake" account that makes it difficult, if not impossible, to trace back the in-world avatar to a real person that can be held accountable for such behavior.

While anonymity can be a feature that is considered acceptable and desirable for certain role-play activities, the necessity for this is not existent when used for educational activities such as those presented in Section 2. Similarly to the previous section, we seek to investigate this further by drawing parallels to social networking sites.

The advancement in technology has brought in efficiency in communication and interaction between people all around the world. However, some of the users are not fully aware of the negatives of certain technology advancements, such as online social 
networking websites. For example, profile cloning is a common activity on many social networking websites, where individuals other than the legitimate owner of a profile create a new profile by copying the original information [Kontaxia et al. 2011]. This is indeed criminal activity on social networking websites that then leads to the creation of a fake profile used to trap legitimate users. The existence of profile cloning/fake accounts is unknown to many users, including vulnerable children.

The issue of fake identities in distributed software systems is anecdotally known across most, if not all, social networking websites, such as Facebook, MySpace, RenRen, Pengyou, and Kaixin. The perhaps most prominent of these is Facebook, which is the fast-growing social networking website with over 901 million users [Yahoo Finance 2012]. Many people nowadays have grown up in a world dominated by online social networks [Medical News Today 2008]. We therefore aim to research further to determine appropriate ways to identify "fake" accounts and in doing so to provide users with protection from cyber predators.

Many social networking websites attract such cyber predators; some, such as Facebook, are specifically prone due to lenient policies (and their implementation) governing identity usage. Facebook's main focus is to enable communication and maintain relationships between users [Dwyer et al. 2007]. An identity is considered to be "fake" if an individual is not using their own identity and therefore cannot be held directly accountable for their actions. Indeed-actions of disguise or deception-seem to be a common activity on social networking websites. Orita and Hada [2009] point out the tension in using pseudonyms rather than real names (on Twitter, in particular): Using pseudonyms imposes greater anonymity, but is it safe to trust a user who does not reveal their real identity? A survey conducted by a consumer reports investigation team has found that one in four users on Facebook lie on their profiles [Gross 2012]. While users may lie on their profiles to conceal certain pieces of information about themselves, this can still be considered as a form of deception, as in doing so users create personas that differ from their real-world identities.

As a first defence measure against identity thefts and attacks, Kontaxia et al. promote more-strict default privacy policies. While this has indeed been introduced to social networks such as Facebook, cyber predators have found other ways to perform such attacks. False names, age, gender, and so on, are some of the key attributes of a fake identity. An example of these attributes used on Facebook is the case of Gemma Barker [O'Donovan 2012]. Gemma Barker, a girl, used three identities to date girls (friends from school) who were using Facebook. She disguised herself as a boy and altered her character to trap the other girls. Her invention of three alter egos shows the ease with which fake identities can be established on Facebook.

Indeed, casual browsing of Facebook shows easily that the use of fake identities is significant, despite Facebook's policy to disallow the use of fake identities [Facebook 2012], especially fake names. A generation of new Facebook users may be involved in a world of lies and deceit. These users are involved in a world where people lie about their identity and also where relationships are quickly disposed of at the click of a mouse [Medical News Today 2008]. This is potentially dangerous, leading many users to be hurt and also to be part of dangerous acts. While Kontaxia et al. [2011] have designed approaches for detecting forged profiles, it does not, for instance, account for online profiles created by users using non-existing information. Having a system in place to determine one's identity through the use of identity credentials would be beneficial for a highly popular social networking website like Facebook.

Therefore, future work in our research will also address this aspect of faking identity and to identify how results of these can be translated to the area of virtual worlds: We seek to extent to which users are able to determine whether an account is real or fake. By evaluating the factors that users depend on to determine whether an account is 
real or fake, we will investigate the feasibility of putting credentials in place to audit a user's identity.

\section{CONCLUSIONS}

To facilitate a situated learning approach, virtual worlds can certainly support students for tasks that allow simulating a challenging but safe environment. We identified that immersion plays a central role in this experience: Appropriate context, for instance, in the setup of a virtual world that links various "local" virtual environments together, becomes a prerequisite to enhance this immersive learning. This raises two questions in particular: which architecture should be used when connecting these virtual environments together and-not unrelated to the technical feasibility-how to identify, avoid, and counter "fake" identities in such an environment so the people behind their avatars can be held accountable for their actions. We have shown how both of these questions are part of current research in the area of online social networks. Answers to these will then fertilize the ground on how similar mechanisms can be fostered to create safe but realistic virtual environments in which teaching can take place.

Indeed, there is a wider issue to be considered here. Given the recent focus on "augmented reality" rather than purely virtual worlds, we would expect in the future a "blended approach" among different types of media, including virtual worlds, OSN, and the "real world." This blur between the real and virtual worlds was already envisaged in 2001 by Okada et al. within the context of environmental education but then was later contextualized within the educational use of Second Life in Conrad et al. [2011]. Charles [2012] most comprehensively identifies a number of areas where the virtual realm tries to fertilize the "real" world and indeed vice versa.

While the parallelism between distributed social networks and virtual worlds might feel somewhat constructed on the technological level, it is obvious that these two domains will show signs of convergence on the social interaction level. Casually browsing Facebook for Second Life already shows a plethora of Second Life avatars as Facebook members and-vice versa-Linden Lab launched their own Community Platform, "My Second Life," in 2011 to support OSN-style interaction.

Technology has to address the issues implied by OSN's and the virtual world and their interaction with reality. This article aims to answer some of these and highlights the need for more research in this area in the future.

\section{REFERENCES}

A. Bielenberg, L. Helm, A. Gentilucci, D. Stefanescu, and H. Zhang. 2012. The growth of diaspora - A decentralized online social network in the wild. In Proceedings of the 2012 IEEE Conference on Computer Communications Workshops (INFOCOM WKSHPS), 13-18.

D. Bowman and R. McMahan. 2007. Virtual reality: How much immersion is enough? IEEE Comput. 40, 7, 2007, 36-43.

S. Buchegger, D. Schióberg, L. H. Yu, and A. Datta. 2009. Peerson: P2p social networking-early experiences and insights. In Proceedings of the 2nd ACM Workshop on Social Network Systems.

D. Carr and M. Oliver. 2009. Second life, immersion and learning. In Social Computing and Virtual Communities. Chapman and Hall. Taylor \& Francis, London, 250-219.

A. Charles. 2012. Interactivity: New Media, Politics and Society. Peter Lang Ltd., 2012.

A. Christopulos and M. Conrad. 2012. Views of 2nd European Immersive Education Summit, Educators on Immersion in Virtual Worlds from Second Life to OpenSim. Paris, France.

M. Conrad. 2011a. Teaching project management in second life. In Multi-User Virtual Environments for the Classroom: Practical Approaches to Teaching in Virtual Worlds, G. Vincenti and J. Braman (Eds.). IGI Global, 2011.

M. Conrad. 2011b. Leaving the lindens: Teaching in virtual worlds of other providers. In Proceedings of Researching Learning in Immersive Virtual Environments (ReLIVE). 
M. Conrad, A. Charles, and J. Neale. 2011. What is my Avatar? - Who is my avatar? The avatar as a device to achieve a goal: Perceptions and implications. In Reinventing Ourselves: Contemporary Concepts of Identity in Virtual Worlds, A. Peachy and M. Childs (Eds.). Springer, UK, 2011.

M. Conrad, D. Pike, P. Sant, and C. Nwafor. 2009. Teaching large student cohorts in second life. In International Conference on Computer Supported Education 2009, J. Cordeiro, B. Shishkov, A. Verbraeck, and M. Helfert (Ed.). INSTICC Press, Lisbon, Portugal, 1, 11-18.

A. Cox, P. Cairns, N. Berthouze, and C. Jennett. 2006. The use of eyetracking for measuring immersion. In Proceedings of the CogSci Workshop: What Have Eye Movements Told Us So Far and What Is Next?

C. Dwyer, S. R. Hiltz, and K. Passerini. 2007.Trust and privacy concern within social networking sites: A comparison of Facebook and MySpace. In Proceedings of the 13th Americas Conference on Information Systems, Colorado.

Facebook. 2012. Disabled Fake Name. Retrieved May 11, 2012 from http://www.facebook.com/help/ 249092175207621.

M. Fischer, T. J. Purtell, R. Chur, and M. S. Lam. 2010. Mr. Privacy: Open and federated social networking using email. In Tech Report, 2010.

D. Gross. 2012. Survey: 1 in 4 users lie on Facebook. Retrieved May 11, 2012 from http://edition. cnn.com/2012/05/04/tech/social-media/facebook-lies-privacy/index.html.

J. Herrington and R. Oliver. 2000. An instructional design framework for authentic learning environments. Educ. Technol. Res. Dev. 48, 3, 23-48.

H. Jaakkola, P. Linna, J. Henno, and J. Makela. 2011. (Social) networking is coming-are we ready? 2011 Proceedings of the 34th International Convention (MIPRO'11), 1133-1139.

A. Kanamgotov, A. Christopoulos, M. Conrad, and S. Prakoonwit. 2012. Immersion in Virtual Worlds-but not Second Life! In Proceedings of the IEEE Cyberworlds Conference.

B. Krishnamurthy and C. Wills. 2009. On the leakage of personally identifiable information via online social networks. In Proceedings of the 2nd ACM SIGCOMM Workshop on Online Social Networks, 2009.

G. Kontaxis, I. Polakis, S. Ioannidis, and E. P. Markatos. 2011. Detecting social network profile cloning. In Proceedings of the 3rd International Workshop on Security and Social Networking.

D. Lee, M. Lim, and S. Han. 2002. ATLAS: A scalable network framework for distributed virtual environments. In Proceedings of the 4th International Conference on Collaborative Virtual Environments. ACM, New York, NY, 47-54.

S. McCloud. 1994. Understanding Comics. HarperCollins.

Medical News Today. 2008. 'Facebook Generation' Faces Identity Crisis. Retrieved November 27, 2011 from http://www.medicalnewstoday.com/releases/113878.php.

E. Moore, T. T. Utschig, K. A. Haas, B. Klein, P. D. Yoder, Y. Ying Zhang, and M. H. Hayes. 2008. Tablet PC technology for the enhancement of synchronous distributed education. IEEE Trans. Learn. Technol. 1,2 (Apr.-Jun.), 2008.

G. O'Donovan. 2012. The Girl Who Became Three Boys. Retrieved August 30, 2012 from http://www.telegraph. co.uk/culture/tvandradio/tv-and-radio-reviews/9459791/The-Girl-Who-Became-Three-Boys-Channel-4review.html.

A. Orita and H. Hada. 2009. Is that really you?: An approach to assure identity without revealing real name online. In Proceedings of the 5th ACM Workshop on Digital Identity Management.

R. Pausch, D. Proffitt, and G. Williams. 1997. Quantifying Immersion in virtual reality. Proceedings of the 24th Annual Conference on Computer Graphics and Interactive Techniques (SIGGRAPH'97).

PMI. 2008. A Guide to the Project Management Body of Knowledge (PMBOK ${ }^{\circledR}$ Guide). Project Management Institute.

S. Seong, J. Seo, M. Nasielski, D. Sngupta, S. Hangal, S. K. Teh, R. Chu, B. Dodson, and M. S. Lam. 2010. PrPl: A decentralized social networking infrastructure. In Proceedings of the 1st ACM Workshop on Mobile Cloud Computing \& Services: Social Networks and Beyond ACM, New York, NY. 8, 1-8, 8

Yahoo Finance. 2012. Number of Active Users at Facebook Over the Years. Retrieve May 11, 2012 from http://finance.yahoo.com/news/number-active-users-facebook-over-years-214600186-finance.html.

C. A. Yeunug, I. Liccard, K. Lu, O. Senevirante, and T. Berners-Lee. 2009. Decentralization: The future of online social networking. In Proceedingso f the W3C Workshop.

Received December 2012; revised February 2013; accepted October 2016 